\title{
Cerâmicas arqueológicas brasileiras: uma revisão de estudos arqueométricos em sítios arqueológicos do Acre, bacia Amazônica e da Região dos Lagos, Rio de Janeiro
}

\section{Alfredo Victor Bellido Bernedo* \\ Rose Mary Latini}

Universidade Federal Fluminense (UFF) Niterói (RJ), Brasil.

*Autor correspondente: alfredobellido@gmail.com

\section{Resumo}

Os estudos de caracterização e classificação de fragmentos cerâmicos baseados na composição química fornecem importantes informações sobre sua origem geográfica. O objetivo deste artigo é apresentar uma revisão dos estudos de caracterização, classificação, proveniência, técnicas de manufatura e datação arqueológica de cerâmicas da Bacia Amazônica; no estado do Acre, norte do Brasil e da Região dos Lagos; estado do Rio de Janeiro, sudeste do Brasil. Estes fragmentos foram estudados por análise por ativação neutrônica instrumental (AANI), datação por termolumescência, espectroscopia Mössbauer, neutrongrafia e radiografia. As diferenças de composição química dessas cerâmicas indicam que estas foram produzidas utilizando-se diferentes argilas e/ou diferentes métodos de fabricação e, os parâmetros de Mössbauer complementam as informações obtidas pela análise por ativação neutrônica, sendo que a AANI fornece informações sobre a origem geográfica e o Mössbauer sobre as técnicas de manufatura. Os estudos de proveniência mostraram que os elementos terras raras são os mais significativos para prover informações sobre a origem geográfica de fragmentos. A média de idade para os sítios do estado do Acre e da região do Rio de Janeiro são de 1950 BP e 300 PB, respectivamente. A neutrongrafia e a radiografia mostram diferentes detalhes de fabricação, cavidades na massa de argila e diferentes temperos. Resultados preliminares mostram que essas técnicas aplicadas no estudo de produção de cerâmicas fornecem informação sobre sua classificação.

Palavras-chave: arqueometria, fragmentos cerâmicos, análise por ativação neutrônica, termoluminescência, espectroscopia Mössbauer, neutrongrafia, radiografia.

\section{Abstract}

Characterization and classification studies of pottery fragments based on chemical composition provide important information about their geographical origin. The aim of this work is to present a review the studies of characterization, classification, provenance, manufacturing techniques and archaeological dating of pottery from the Amazon basin in the State of Acre, northern Brazil, and from the Região dos Lagos, State of Rio de Janeiro, southeastern Brazil. These pottery fragments have been studied using instrumental neutron activation analysis (INAA), thermoluminescence dating, Mössbauer spectroscopy and neutron and X-Ray radiography. The classification differences in ceramic chemical composition suggest that they were made using different clay and/or 
different methods of fabrication and the Mössbauer parameters complement the interpretation obtained by INAA for ceramics classification studies since the INAA provides information about the geographic origin and Mössbauer on manufacturing techniques. For the provenance studies the rare earth elements were the most significant elements for giving informations on the geographical origin of the different fragments. The average date for the sites from State of Acre and Region of Rio de Janeiro are 1950 BP and 300 BP, respectively. The neutron and $X$-Ray radiography show different manufacturing details, cavities in the clay body and different temper. The preliminary results show these techniques applied in pottery production studies provide important information about classification of ceramics.

Keywords: archaeometry, pottery sherds, neutron activation analysis, thermoluminescence, Mössbauer spectroscopy, neutron, X-Ray radiography.

\section{INTRODUÇÃO}

Interações culturais entre comunidades são algumas das importâncias reveladas pela classificação arqueológica de cerâmicas. Neste sentido, as cerâmicas são objetos de grande valor arqueológico por causa de sua constituição física e mineralógica que as fazem resistentes às condições do tempo e do meio circundante, podendo fornecer informações a respeito de trajetórias (procedências) e características culturais entre grupos e dentro de grupos de povos que habitaram determinada região. Cerâmicas são confeccionadas com argilas que, muitas vezes, são misturadas com "temperos", materiais utilizados para melhorar a plasticidade, antes de serem queimadas. Estes temperos podem ser conchas, cascas de árvores, areia, ou restos de cerâmicas, dentre outros. Estas são classificadas e catalogadas por arqueólogos com base em atributos como cor, forma, tipo de material, e tempero utilizado, dentre outros.

Nos grupos de argilominerais, as espécies mineralógicas e a respectiva composição química das argilas apresentam variações causadas pelas diferentes condições geológicas de formação e em função do grau de substituições isomórficas ou troca por similaridade e cátions trocáveis. A importância dos elementos traços na composição de cerâmicas reside no fato de que estas refletem as características de composição química das argilas que a constituem. Considera-se estatisticamente improvável que a concentração de vários elementos traços de argilas de uma determinada localidade coincida quantitativamente com a de argilas de outras procedências (Travesi 1975).

Assim, a composição química de cerâmicas, principalmente a de elementos terra raras (grupo dos lantanídeos),

\section{1 Áreas de Estudo}

As cerâmicas arqueológicas em estudo pelo grupo são oriundas da bacia Amazônica, mais especificamente da reflete as características da composição geoquímica original das argilas que as constituem, o que confere uma "impressão digital" a cada um dos fragmentos cerâmicos analisados por técnicas multielementares. Ao lado da composição química, as características físicas do material podem fornecer informações a respeito da tecnologia de fabricação de cerâmicas, como a temperatura de queima e a estrutura interna das cerâmicas. E, a correlação de todos estes parâmetros, permite uma melhor caracterização e classificação destas.

Este artigo tem por objetivo apresentar os resultados dos estudos de caracterização, classificação, análise de procedência, manufatura e datação de cerâmicas arqueológicas da Bacia Amazônica, no Acre e da Região dos Lagos no Rio de Janeiro, que foram realizados por pesquisadores do Grupo de Pesquisa em Arqueometria ligados ao Laboratório de Química Nuclear e Radioquímica/UFF, utilizando-se diferentes técnicas analíticas nucleares associadas à análise estatística de multivariáveis. A escolha das áreas de estudo está relacionada com a abundância de fragmentos cerâmicos e a presença de cerâmicas intactas, material funerário e utilitário.

Os objetivos dos estudos na bacia Amazônica do Acre foram de caracterizar e classificar as cerâmicas da região; comparar estes resultados com a classificação feita por arqueólogos, identificar a procedência, os parâmetros de temperatura de queima e de manufatura das cerâmicas e estabelecer o período de ocupação dos sítios circulares e o sentido de ocupação destes na bacia Amazônica do Acre.

$\mathrm{Na}$ Região dos Lagos, Rio de Janeiro, os estudos tiveram por objetivos a caracterização, classificação, datação e identificação de parâmetros de manufatura das cerâmicas.

bacia do rio Purus, no estado do Acre e dos municípios de Araruama e Búzios na Região dos Lagos no Rio de Janeiro 


\subsubsection{Bacia Amazônica - Estado do Acre}

Os solos da região estudada no Acre são de origem sedimentar, sendo constituídos predominantemente por solos podzólico vermelho-amarelo com presença de solos hidromórficos gleyzados, situados nas margens de rios e igarapés. A vegetação natural é composta basicamente de floresta tropical densa e aberta, caracterizada por heterogeneidade de espécies. A rede hidrográfica da região faz parte da bacia Amazônica, sendo formada pelas bacias dos rios Juruá e Purus, afluentes da margem direita do Rio Solimões (Projeto Radam v.19 apud Latini 1998). As cerâmicas em estudo foram encontradas em sítios arqueológicos da bacia do rio Purus, no estado do Acre (Figura 1).

Nas cerâmicas da bacia Amazônica é comum a utilização de cauxi, um espongiário de água doce, e de cariapé, uma casca de árvore como "temperos" (Costa et al. 2009). Assim, estes são temperos comuns nas cerâmicas em estudo, além de outros, como areia, cerâmica moída e carvão.

Acredita-se existir no Acre duas Tradições Culturais (Dias e Carvalho 1981, 1988, Meggers 1988): uma na bacia do rio Juruá e outra na bacia do rio Purus. Tradição cultural pode ser entendida como fases arqueológicas que compartilham de um mesmo conjunto de técnicas diagnosticadas pela forma e decoração das cerâmicas (pintura, formato, etc.) e fase arqueológica, por sua vez, como a representação de uma unidade social similar, isto é, de comunidades que interagem mais intensamente entre si (Meggers 1990 apud Latini 1998). De acordo com Dias e Carvalho (1981) e Meggers (1988), a Tradição Cultural sobre a bacia do Rio Purus é denominada Quinari e as fases arqueológicas Iquiri, Quinari, Xapuri, Jacuru e Iaco.

Alguns sítios arqueológicos no Acre apresentam estrutura de terra circular em forma de valeta e muretas, onde é encontrada grande quantidade de fragmentos de cerâmica. O conjunto valeta e muretas é interceptado por um "ramal" ou caminho que leva a um rio mais próximo (Figura 2). Estas estruturas possuem diâmetros que variam de 50 a 350 metros e a sua finalidade é desconhecida por arqueólogos. Os sítios arqueológicos com estrutura de terra circular identificados até o momento no Acre são Los Angeles, Xipamanu I, Xipamanu II (próximos ao município de Xapuri), Alto Alegre (próximo à capital Rio Branco) e Lobão (próximo à cidade Sena Madureira). A datação das cerâmicas encontradas nessas estruturas comprova a sua antiguidade e a sua relação com a pré-história local (Latini 1998). Algumas destas estruturas podem ser visualizadas por imagens de satélite e fotografias aéreas (Nícoli 2000). Outras estruturas de terra com diferentes formas geométricas foram também identificadas nesta região.

As amostras de cerâmicas em estudos foram coletadas em sítios arqueológicos da região com e sem estrutura de terra circular, a maioria delas coletadas pelo arqueólogo Ondemar Dias Junior e sua equipe. Entretanto, as cerâmicas utilizadas nos estudos de datação foram coletadas pelos autores e pelos pesquisadores Marcos Vinicius Neves e Ieda Nícoli, nos sítios arqueológicos com estrutura de terra circular, em diferentes profundidades.

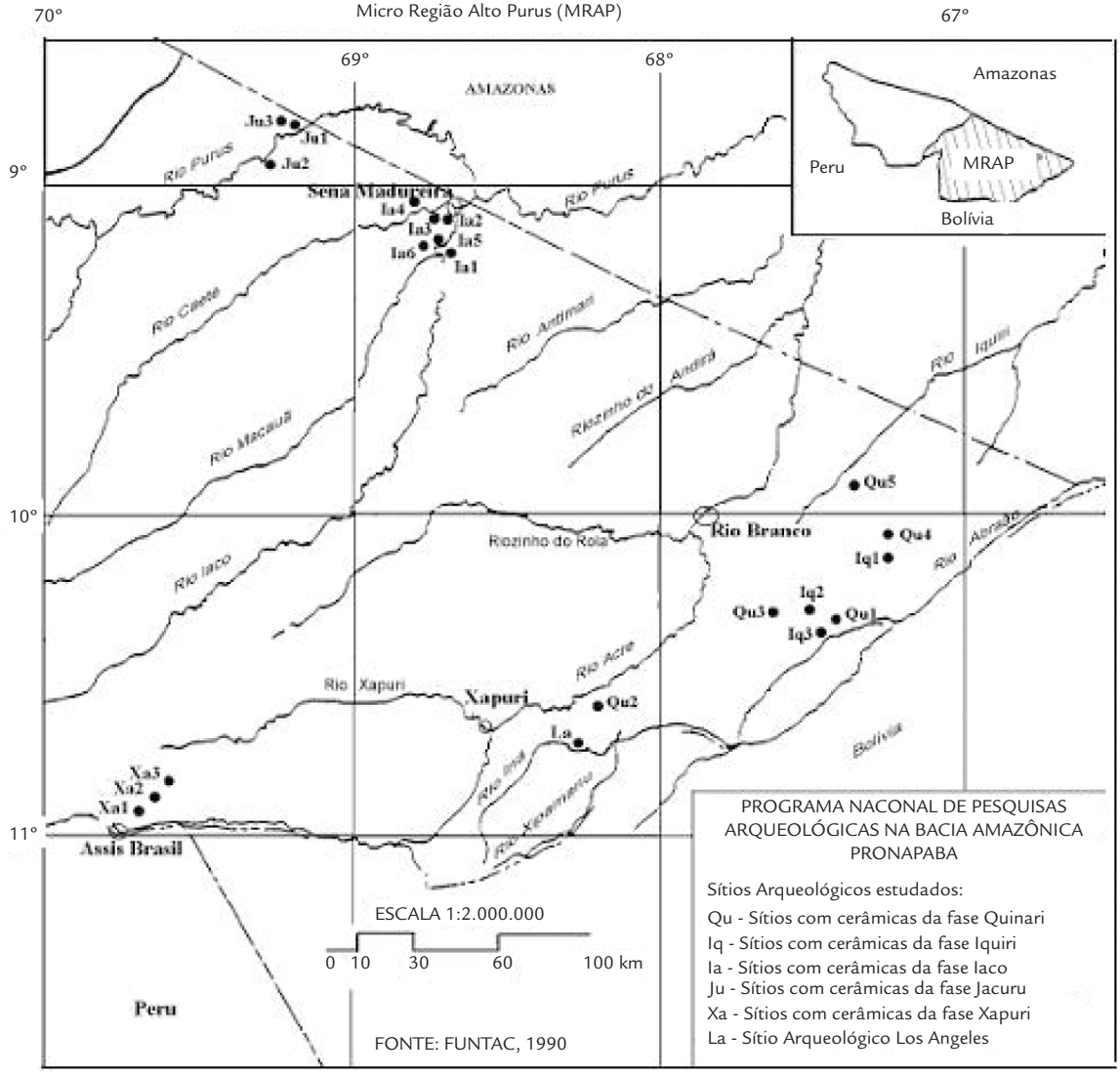

Figura 1

Área de estudo na bacia do rio Purus no estado do Acre, mostrando a localização dos sítios arqueológicos estudados.

(Modificado de Meggers 1998). 


\subsubsection{Região dos Lagos, Rio de Janeiro}

Os sítios arqueológicos estudados na Região dos Lagos do Rio de Janeiro são Boa Vista, Serrano, Morro Grande e São José, no município de Araruama, e Una, no município de Búzios.

Os sítios arqueológicos de Morro Grande encontramse atualmente em área urbana e, apesar de parcialmente destruídos pela urbanização, alguns deles ainda apresentam estruturas arqueológicas intactas e peças cerâmicas bem conservadas (Buarque 1996).

Algumas das cerâmicas em estudo são oriundas do salvamento realizado na ocasião da construção da rodovia

\section{METODOLOGIA}

Nas duas regiões, através da técnica de análise por ativação com nêutrons foi possível identificar 21 concentrações de elementos químicos, que associada à análise estatística de multivariáveis, permitiu classificar e comparar a classificação química com a arqueológica; levando a pré-classificação arqueológica, feita a partir de macro diferenciações, para uma classificação a nível ordenado de composição química
Via Lagos $\left(22^{\circ} 48^{\prime} 2.9^{\prime}\right.$ S, $\left.42^{\circ} 27^{\prime} 52^{\prime} \mathrm{W}\right)$, em conjunto com a equipe do Museu Nacional da Universidade Federal do Rio de Janeiro. Cerâmicas nesta região são também observadas nos últimos níveis de ocupação de sambaquis. Acredita-se que o desaparecimento de grupos pescadores-coletores-caçadores em algumas regiões pode estar associado à chegada de grupos ceramistas. As cerâmicas coletadas nestes sítios foram identificadas como pertencente aos grupos arqueológicos Una e Tupinambá (Tenório 1999, Gaspar 1997). elementar; identificar diferenças entre cerâmica utilitária e funerária e localizar a procedência destas cerâmicas.

O estabelecimento do período de fabricação destas cerâmicas foi realizado pela técnica de datação por termoluminescência e as técnicas de Mössbauer, neutrongrafia e radiografia foram utilizadas para identificação de parâmetros de manufatura.

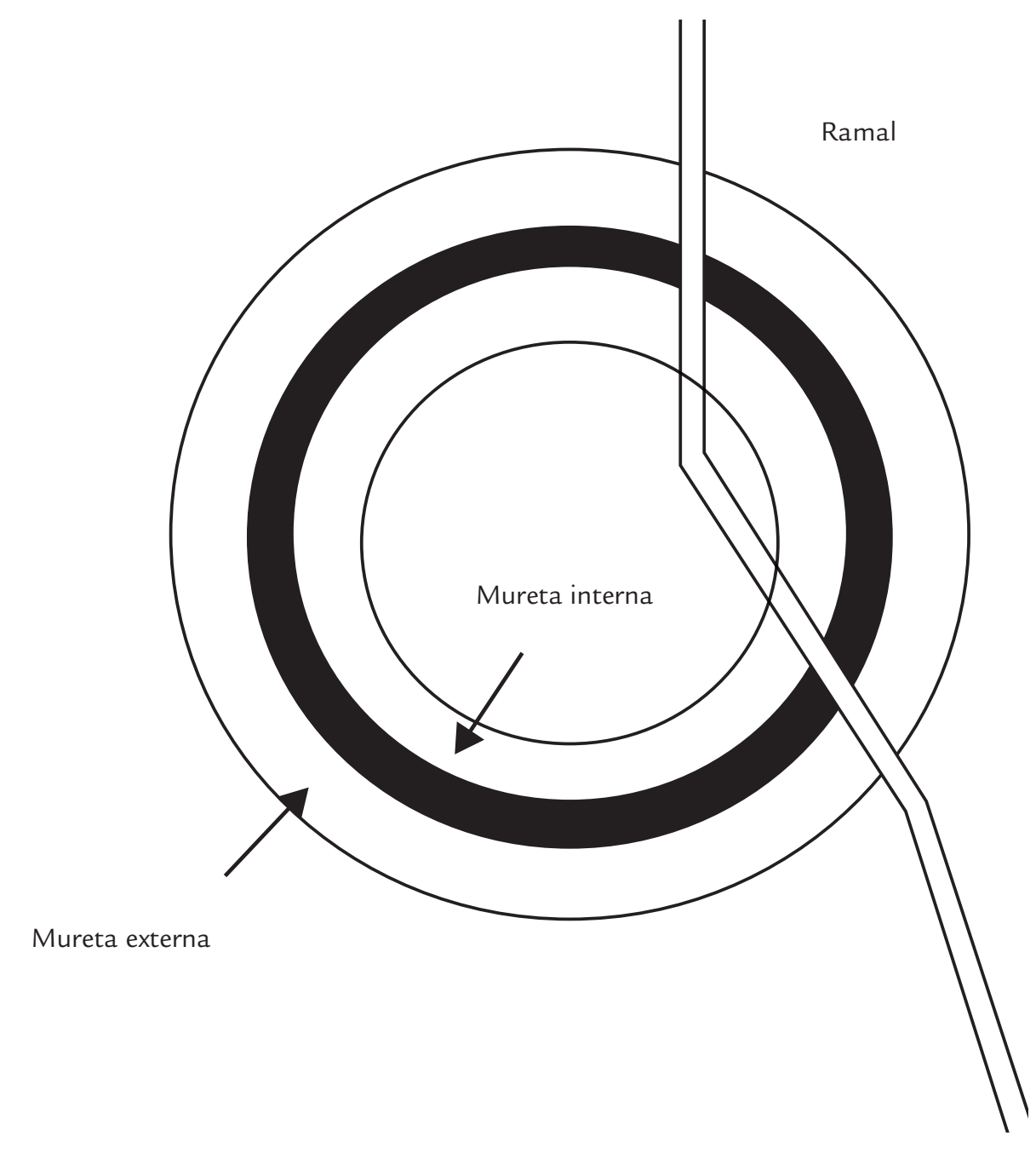

Representação genérica de um sítio arqueológico com estrutura de terra circular na região estudada. 


\subsection{Análises químicas por Ativação Neutrônica}

A análise por ativação neutrônica (AAN), pelo método relativo, foi utilizada para caracterização do material arqueológico, obtida pela análise química quantitativa multielementar dos fragmentos de cerâmicas. O padrão de referência foi o Podmore Red Clay, padrão de argila confeccionado no Laboratório de Química Nuclear da Universidade de Manchester, Inglaterra. Duzentos miligramas de cada uma das amostras foram irradiadas no reator de pesquisa IEA-R1 do Instituto de Pesquisa Energéticas e Nucleares (IPEN/SP), com um fluxo de nêutrons térmicos da ordem de $10^{12}$ nêutrons $/ \mathrm{cm}^{2} / \mathrm{s}$. A ativação das amostras foi realizada em duas etapas de acordo com a meia-vida dos radionuclídeos medidos. Foram considerados 21 elementos químicos livres de interferentes espectrais e com

\subsection{Datação por Termoluminescência}

Para a datação por termoluminescência (TL), fragmentos cerâmicos e sedimentos circundantes foram coletados e imediatamente guardados em sacos plásticos pretos, para prevenir exposição à luz e perda de umidade, a qual foi posteriormente determinada para correção da taxa de dose anual.

A medida do sinal de TL foi feita em grãos de quartzo. Uma camada de aproximadamente $2 \mathrm{~mm}$ de espessura foi retirada de cada um dos lados dos fragmentos de cerâmica, a fim de se eliminar possíveis contaminantes, além de facilitar o cálculo da dose anual no que diz respeito a contribuição da radiação a proveniente do sedimento circundante (MacKeever 1985, Aitken 1985). As amostras foram pulverizadas e a fração com grãos médios de $100 \mathrm{~mm}$ foi selecionada. A confirmação da presença de quartzo nas amostras foi feita por difratometria de raios-X, no Instituto de Engenharia Nuclear (Instituto de Engenharia Nuclear-IEN/RJ).

As idades das cerâmicas foram determinadas pela razão entre a dose total acumulada (paleodose) e a dose

\subsection{Mössbauer}

Para determinação de parâmetros hiperfinos, a análise de espectros de Mössbauer do ${ }^{57} \mathrm{Fe}$ foi realizada à temperatura ambiente utilizando-se aproximadamente $200 \mathrm{mg}$ de amostra (Munayco 2011). Foram analisados noventa e quatro fragmentos cerâmicos pertencentes ao sítio Los

\subsection{Neutrongrafia e Radiografia}

Estas técnicas, não destrutivas, foram utilizadas com o objetivo de visualizar as características internas das cerâmicas sem danificá-las. Para neutrongrafias diferentes fragmentos cerâmicos foram submetidos por 3 minutos a um fluxo de nêutrons da ordem de $10^{5}$ nêutrons $/ \mathrm{cm}^{2} / \mathrm{s}$ no reator de pesquisa Argonauta no Instituto de Engenharia Nuclear da Comissão Nacional de Energia Nuclear. As ima- erros menores do que $5 \%$. Aproximadamente 300 amostras foram analisadas, por esta técnica, nas duas regiões.

A detecção da radiação gama oriunda das amostras ativadas foi feita por meio de detector HPGe cuja resolução é de $1,83 \mathrm{keV}$ para o fotopico de energia de $1332 \mathrm{keV}$ do ${ }^{60} \mathrm{Co}$. A eletrônica associada consistiu em um amplificador e um analisador multicanal de 4096 canais Ortec/Maestro Spectrum 92X. Para análise dos espectros e cálculo das concentrações dos elementos químicos utilizou-se o programa GRGAN, considerando apenas os raios gamas livres de interferentes (Gilmore 1983). As contagens dos radionuclídeos de meia-vida curta foram realizadas no IPEN/SP e os de meia-vida longa no Instituto de Química/UFF.

de radiação anual. A paleodose foi determinada no Centro Brasileiro de Pesquisas Físicas - CBPF, a partir da variação da intensidade de TL, em cinco temperaturas diferentes, contra as doses aditivas que foram de 4, 8, 12, 16 e $20 \mathrm{~Gy}$. A dose anual foi calculada a partir da contribuição das radiações $\mathrm{a}, \mathrm{b}$ e $\mathrm{g}$ oriundas dos radionuclídeos naturais, ${ }^{238} \mathrm{U},{ }^{232} \mathrm{Th}$ e ${ }^{40} \mathrm{~K}$, determinados por AAN, presentes nas cerâmicas e nos sedimentos circundantes e da contribuição da radiação cósmica (Latini 1998). No cálculo de dose anual levou-se em consideração correções de dose devido à atenuação da radiação b e à umidade presente nas cerâmicas e sedimentos circundantes. As incertezas associadas foram decorrentes da determinação dos radionuclídeos presentes no material. Por não ter sido possível determinar a dose anual local, nas idades obtidas, consideramos que não houve perdas de ${ }^{222} \mathrm{Rn}$ e ${ }^{220} \mathrm{Rn}$ nas cerâmicas e nos sedimentos circundantes (idades mínimas) e a perda total de radônio (idade máxima).

Angeles e aos sítios das fases arqueológicas Quinari, Iquiri e Iaco. O tempo de contagem dos espectros foi em torno de 48 horas e os procedimentos de fitting foram realizados no software Normos 95. As análises foram feitas no Centro Brasileiro de Pesquisas Físicas (CBPF).

gens foram obtidas pela técnica de processamento digital, usando-se um "image plate" (Bellido et al. 2011b). Para as radiografias as amostras foram irradiadas com Raios-X no equipamento Feinfocus Model FX100 e as imagens foram obtidas pelo Flat Panel GE IT Model DXR 250V no Laboratório de Instrumentação Nuclear (LIN) da Coordenação de Pós-Graduação e Pesquisa de Engenharia- COPPE/UFRJ. 


\section{RESULTADOS E DISCUSSÃO}

Os dados obtidos pelas diferentes técnicas analíticas foram utilizados no tratamento estatístico de multivariáveis. A análise de discriminantes, de conglomerados e a análise de componentes principais foram usadas no estudo de classificação e ordenação das variáveis (concentrações de elementos químicos), utilizando-se o pacote estatístico SPSS (Statistical Package for the Social Sciences). Inicialmente os dados foram normalizados pela transformação z-scores que gerou novas variáveis, com média zero e desvio padrão igual a um. $\mathrm{Na}$ análise de conglomerados, a distância Euclideana foi utilizada como medida de distância, e fez-se uso da técnica hierárquica de conglomerados na subrotina Ward (variância mínima). A análise de componentes principais foi utilizada para estudar a correlação múltipla entre as variáveis nos grupos formados.

\subsection{Estudos de Caracterização, Classificação e Procedência}

Os elementos químicos determinados por AAN, nos fragmentos cerâmicos e nas argilas foram $\mathrm{Ce}, \mathrm{Co}, \mathrm{Cr}, \mathrm{Cs}, \mathrm{Dy}, \mathrm{Eu}, \mathrm{Al}, \mathrm{Fe}$,
Hf, Lu, Mn, Na, Rb, Sb, Sc, Sm, Ta, Th, Ti, U e V.Todos os elementos foram utilizados nos estudos de classificação e procedência.

\subsubsection{Bacia Amazônica no Acre}

Nos estudos de classificação, inicialmente, foi verificada a validade da classificação feita pelos arqueólogos utilizando-se a análise de discriminantes, tendo sido identificado uma concordância de $70 \%$, em média, entre os resultados obtidos a partir da composição química e a pré-classificação arqueológica definida em fases arqueológicas (Latini 1998; Latini et al. 2001). A Tabela 1 apresenta um resumo do resultado desta análise.

Com os estudos de classificação foram identificadas diferenças na composição química entre as cerâmicas dos sítios arqueológicos com estrutura de terra circular e os sem estrutura circular (Latini 1998).

A Figura 3 mostra o dendograma obtido, a partir da análise de conglomerados, para as amostras dos sítios arqueológicos Alto Alegre, Lobão e Xipamanu I, todos os sítios com estruturas circulares (Bellido et al. 2007).

Observam-se dois grupos distintos. O grupo 1 é subdivido em dois grupos ( $1 \mathrm{~A}$ e $1 \mathrm{~B}$ ) e o grupo 2 engloba apenas amostras do sítio arqueológico Alto Alegre caracterizando assim, um grupo distinto dos demais. A caracterização de cada um dos subgrupos foi realizada pela verificação do efeito de diluição e pela análise de componentes principais. A verificação do efeito de diluição indica que nos subgrupos 1A e 1B não existem diluições entre eles, sendo, portanto, subgrupos distintos. Para o grupo 2 o estudo de diluição apontou que a formação dos três subgrupos foi devida a

diluição do subgrupo $2 \mathrm{~A}$ em relação aos subgrupos $2 \mathrm{~B}$ e $2 \mathrm{C}$, que são quimicamente bastante semelhantes. Observase ainda que no subgrupo $2 \mathrm{~B}$ é diferenciado apenas pelos elementos U, K e Cr e, o subgrupo 2C somente pelo Fe. Pela análise de componente principal identificou-se que os elementos químicos que mais contribuíram para a formação a formação do subgrupo 1A foram: Ce, Cr, Dy, Eu, Fe, K, La, $\mathrm{Lu}, \mathrm{Th}, \mathrm{Sc}, \mathrm{Sm}, \mathrm{U}$ e Yb. Para o subgrupo 1B: Co, Fe, Hf, K, $\mathrm{Lu}, \mathrm{Na}$, Th, Ta, U, e Yb. No subgrupo 2A: CE, Co, Eu, Fe, Hf, K, La, Lu, Mn, Rb, SC, SM, Ta e Yb. No subgrupo 2B: Cr, Hf, K, La, Lu, Th, Rb, SC, Ta, U e Ub. No subgrupo 2C: Fe, Hf, La, Lu, Th, Rb, SC, Ta e Yb. Assim, as maiores diferenças entre os grupos e subgrupos são devidas as correlações internas (Nícoli 2000).

Em relação ao grupo 1, observa-se uma mistura de amostras dos sítios Xipamanu I e Lobão. O agrupamento em dois subgrupos seja talvez devido a diferenças de tecnologia de fabricação, pois as amostras que constituem o grupo 1A são fragmentos de urnas e as do grupo 1B não foram identificadas como provenientes de urnas. Estudos anteriores indicam que cerâmicas utilitárias são quimicamente diferentes de cerâmicas funerárias causando assim uma separação no dendograma (Latini et al. 1999).

No que se refere à composição química das cerâmicas dos sítios circulares estudados, apesar dos sítios arqueológicos Xipamanu I e Alto Alegre estarem geograficamente mais

Tabela 1

Resumo da Análise de Discriminantes.

\begin{tabular}{|c|c|c|c|c|c|}
\hline 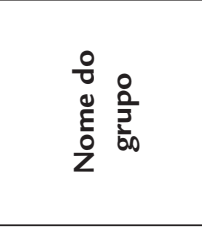 & 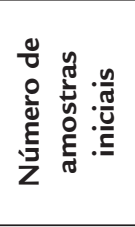 & 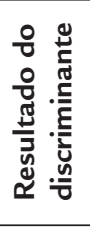 & 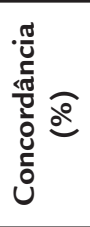 & 들 & 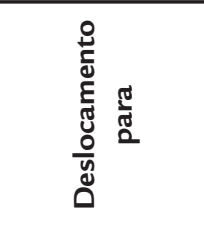 \\
\hline Iquiri (Iq) & 18 & 15 & 83 & 11 & $\mathrm{Qu}$ \\
\hline Quinara (Qu) & 20 & 15 & 75 & 13 & lq e La \\
\hline Iaco (la) & 22 & 7 & 32 & 4 & $\mathrm{Qu}, \mathrm{Ja}, \mathrm{Xa}$ e La \\
\hline Xapuri (Xa) & 17 & 15 & 88 & 10 & $\mathrm{la}$ \\
\hline Jacuru (Ja) & 11 & 7 & 64 & 3 & la e Xa \\
\hline SALA (La) & 74 & 40 & 54 & 29 & $\mathrm{Qu}, \mathrm{Ja}, \mathrm{Xa}$ e La \\
\hline
\end{tabular}


próximos um do outro, a análise do dendograma mostra que suas cerâmicas não são similares quimicamente, sugerindo que estas possuem tecnologias de fabricação diferentes. Por outro lado, apesar dos sítios Xipamanu I e Lobão estarem geograficamente distantes um do outro, possuem cerâmicas com similaridades tecnológicas (Nícoli 2000).

Em outros estudos de classificação foram utilizadas um total de 16 variáveis obtidas por espectroscopia Mössbauer, juntamente com os dados obtidos por ativação neutrônica para cerâmicas do sítio Arqueológico Los Angeles e cerâmicas das fases arqueológicas Iaco, Iquiri e Quinari. Os resultados indicam que os parâmetros de Mössbauer complementam a interpretação obtida com os dados da AAN, pois incorpora características das técnicas de manufatura, como a temperatura de queima no momento da confecção da cerâmica que influencia nas quantidades dos elementos químicos nela

\subsubsection{Região dos Lagos, Rio de Janeiro}

No Rio de Janeiro, o trabalho realizado por Latini et al. (1999) com cerâmicas encontradas nos sítios arqueológicos São José e Morro Grande em Araruama mostrou que há diferença de composição química entre os fragmentos que apresentam algum tipo de decoração plástica (corrugado, escovado, etc.), os sem decoração e as cerâmicas funerárias. Diferenças de composição química entre as cerâmicas dos sítios Serrano e Bela Vista também foram identificadas (Vinagre Filho et al. 2005a). As diferenças na composição química são relacionadas com as quantidades de cada um dos elementos químicos presentes nas cerâmicas e com as correlações internas de cada grupo.

Os resultados obtidos indicam a formação de cinco grupos de cerâmicas de acordo com as suas similaridades de presentes (Bellido et al. 2011a, Munayco 2011). A Figura 4 mostra a formação de quatro grupos distintos.

Estudos estatísticos posteriores, realizados com os dados de concentração obtidos por neutrônica e com os parâmetros de Mössbauer, indicam que os elementos terras raras (ETR) são variáveis discriminantes nos estudos de classificação de cerâmicas arqueológicas. Isto é, utilizando-se as concentrações apenas dos ETR já é possível classificá-las (Bellido et al.2011a).

No sítio arqueológico Los Angeles, que apresenta estrutura circular, foram também realizados estudos de procedência das cerâmicas. Identificou-se que algumas das cerâmicas deste sítio foram confeccionadas com argila branca, chamada pelos moradores da região de Tabatinga, das margens do rio Ina, localizado à uma hora de caminhada do sítio (Latini et al. 2004).

composição química. Na Figura 5 verifica-se que foi formado um grupo pertencente ao sítio São José (F) que engloba cerâmicas sem corrugação, dois grupos de cerâmicas do sítio arqueológico Bela Vista (BVC), um grupo que engloba amostras do sítio arqueológico Serrano (SSC) e do sítio arqueológico São José (F), e um último grupo formado por cerâmicas do sítio arqueológico São José e fragmentos de urnas funerárias dos sítios arqueológicos São José e Morro Grande (2005)

Observa-se que as cerâmicas do Sítio arqueológico São José praticamente se encontram distribuídas na maioria dos grupos, o que pode ser explicado pela proximidade dos sítios estudados. Para análise de procedência das cerâmicas foi realizado um

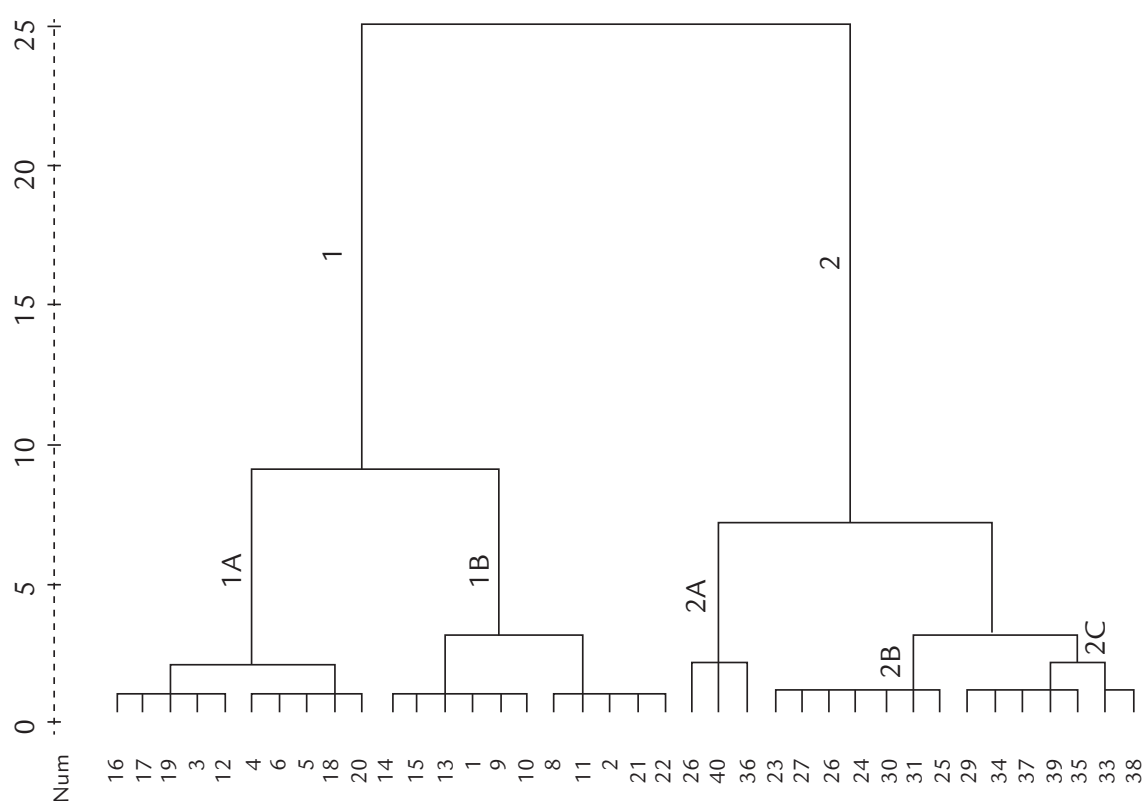

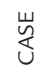

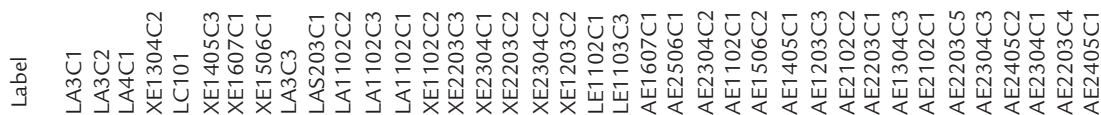

Figura 3

Dendograma com fragmentos cerâmicos dos sítios arqueológicos com estruturas circulares Alto Alegre, Lobão e Xipamanu I, Acre. 
Figura 4

Classificação de cerâmicas de sítios arqueológicos da bacia Amazônica no Acre utilizando variáveis análise por ativação neutrônica e de Mössbauer.

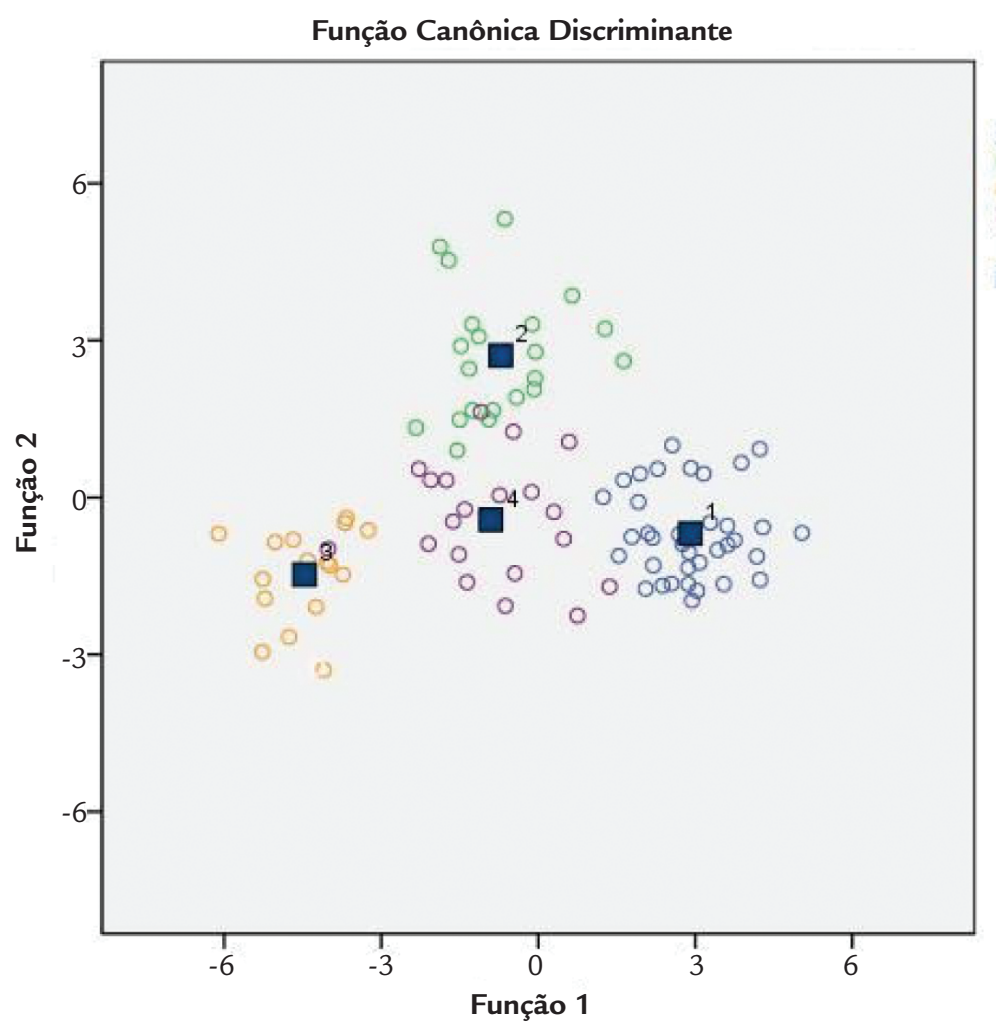

\section{Grupos}

1 Los Angeles

O laco

3 Iquiri

4 Quinari

Centroide
Figura 5

Dendograma com dados de análises químicas de cerâmicas e argilas de sítios arqueológicos da região dos Lagos, Rio de Janeiro.

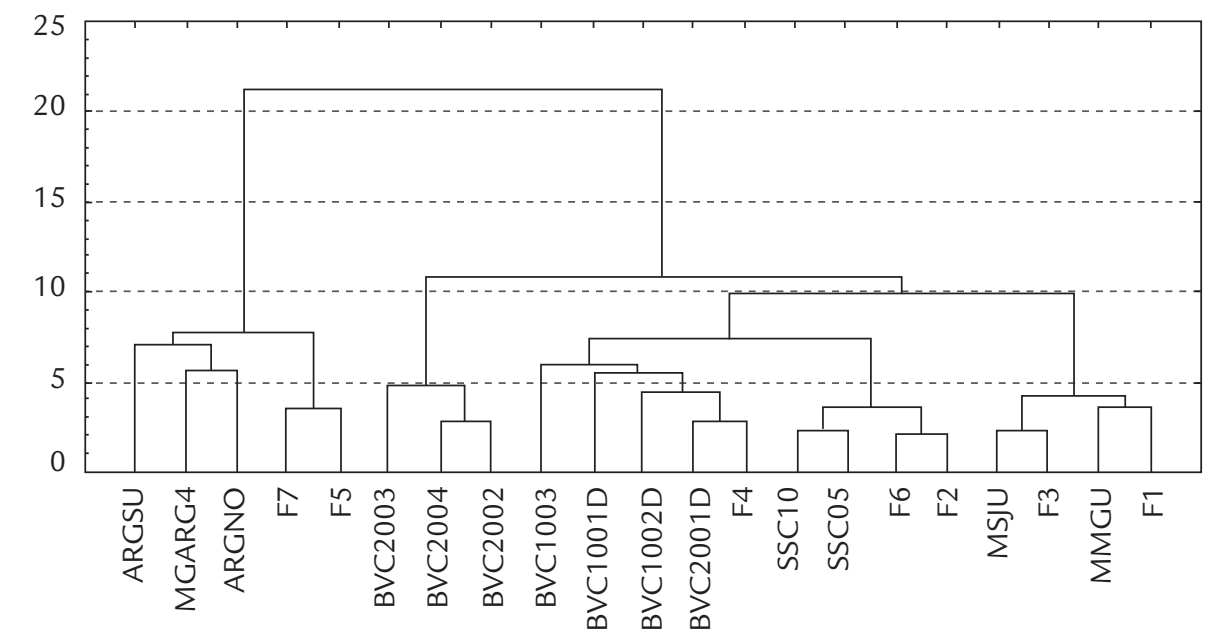

ARG: Argila; BV: Sítio Bela Vista; SSC: Sítio Serrano; F e MSJU: Sítio São José; MMGU: Morro Grande. estudo comparativo entre os grupos definidos pela análise de conglomerados e as argilas. Os resultados indicam que as cerâmicas que apresentam composição química mais semelhante com as argilas coletadas (ArgNo e ArgSu) são as cerâmicas sem corrugação encontradas no sítio arqueológico São José (F5 e F7) integrantes do Grupo 1 (Vinagre Filho et al. 2005b). 


\subsection{Datação por Termoluminenscência}

\subsubsection{Bacia Amazônica no Acre}

Nos sítios arqueológicos com estrutura de terra circular os resultados sugerem que a idade média de ocupação nos sítios arqueológicos Los Angeles, Xipamanu I, Alto Alegre e Lobão é de 1620, 2380, 1976 e 1835 BP, respectivamente, sendo que no sítio Lobão foi obtida apenas uma idade (Latini 1998, Nicole 2000). Na Tabela 2 podem ser vistos as idades obtidas para esses sítios.

A comparação das idades mais antigas e mais recentes de cada um dos sítios indica contemporaneidade, em um

\subsubsection{Região dos Lagos, Rio de Janeiro}

As idades de cerâmicas dos sítios arqueológicos São José, Morro Grande e Bela Vista foram de 282, 311 e $310 \mathrm{BP}$, respectivamente, indicando que estes sítios são recentes e foram contemporâneos, embora (Latini 1998, determinado período, entre os quatro sítios arqueológicos (Figura 6).

No sítio arqueológico Los Angeles as idades determinadas para as amostras coletadas num corte escavado sobre a mureta externa indicam um comportamento estratigráfico invertido até o nível 40-50 cm identificado como nível de ocupação. Essas idades são coerentes com as analisadas pelo método radiométrico de ${ }^{14} \mathrm{C}$, realizadas no Laboratório Beta Analytical para o mesmo sítio, obtidas por Latini (1998).

Latini et al. 1999, Vinagre Filho 2005a). No momento, estudos de procedência, datação e investigação da interface de grupos ceramistas e sambaquieiros, no sítio Una, estão em andamento.

\begin{tabular}{lccc}
\hline Amostra & $\begin{array}{c}\text { Profundidade } \\
(\mathbf{c m})\end{array}$ & $\begin{array}{c}\text { Idade Mínima } \\
*(\mathbf{B P})\end{array}$ & $\begin{array}{c}\text { Idade Máxima } \\
* *(\mathbf{B P})\end{array}$ \\
\hline 2LA51 & $0-10$ & 1788 & 2455 \\
\hline 2LA52 & $10-20$ & 1286 & 1823 \\
\hline 2LA54 & $30-40$ & 1220 & 1682 \\
\hline 2LA551 & $40-50$ & 902 & 1182 \\
\hline 2LA552 & $40-50$ & 2322 & 3032 \\
\hline 2LA5060 & $50-60$ & 1625 & 2210 \\
\hline 3LA57 & $10-20$ & 2147 & 2894 \\
\hline 16LA28 & $20-30$ & 1621 & 2103 \\
\hline 16LA30 & $20-30$ & 1662 & 2170 \\
\hline XE2203C1 & $20-30$ & 2623 & 4052 \\
\hline XE2203C2 & $20-30$ & 2050 & 3546 \\
\hline XE2203C3 & $20-30$ & 1936 & 3211 \\
\hline XE2304C2 & $30-40$ & 2908 & 2220 \\
\hline LBC1U1 & 110 & 1835 & 2986 \\
\hline AE2203C3 & $20-30$ & 1804 & 2970 \\
\hline AE2203C5 & $20-30$ & 1873 & 3543 \\
\hline AE2405C1 & $40-50$ & 2250 &
\end{tabular}

Tabela 2

Idades obtidas por termoluminescência para as amostras dos sítios arqueológicos Los Angeles, Xipamanu I, Lobão e Alto Alegre, Acre.

*Idade calculada considerando-se que não houve perdas de radônio. **Idade calculada considerando-se perda de $100 \%$ de radônio. Profundidade referida na tabela é aquela de coleta de amostra em relação à superfície; LA: Los Angeles; XE: Xipamanu I; LB: Lobão; AE: Alto Alegre.

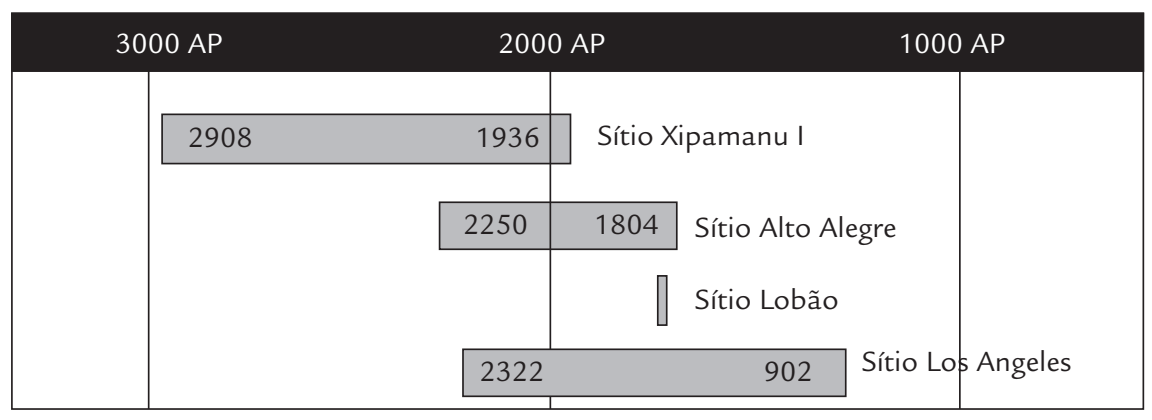

Figura 6

Vista Cronológica dos períodos de ocupação dos sítios arqueológicos Xipamanu I, Alto Alegre, Lobão e Los Angeles, Acre. Idades mínimas em BP (Nícoli 2000). 


\subsection{Parâmetros de Manufatura}

Técnicas como espectroscopia Mössbauer, tomografia e neutrongrafias têm sido utilizadas para se obter informações a respeito de tecnologia de fabricação de cerâmicas arqueológicas, permitindo-se conhecer o tipo de ambiente de queima utilizada na sua manufatura e também a sua estrutura interna, isto é, se ela foi construída utilizando "roletes" ou "paletado". Roletes referem-se à técnica de manufatura de cerâmica que utiliza argila moldada em forma de rolos sobrepostos uns sobre os outros e, paletado refere-se à técnica de manufatura que utiliza uma massa homogênea de argila na confecção do vaso de cerâmica (Parrelada 2010).

Com os estudos de espectroscopia Mössbauer realizado com cerâmicas do sítio Arqueológico Los Angeles e cerâmicas das fases arqueológicas Iaco, Iquiri e Quinari, todas da bacia Amazônica no Acre, verificou-se que as cerâmicas foram fabricadas tanto em ambiente oxidante como em ambiente redutor, no momento da queima (Bellido et al.2011a, Munayco 2011).
Em relação aos estudos relacionados à estrutura interna das cerâmicas já foi possível, através da neutrongrafia, identificar diferenças de manufatura com cerâmicas da bacia Amazônica no Acre construídas em forma de roletes sobrepostos e/ou paletados (Bellido et al. 2011b, 2011c). $\mathrm{Na}$ neutrongrafia de cerâmicas construídas pela técnica de roletes podem ser observados traços horizontais da junção de tais roletes, formando camadas sobrepostas (Figura 7a). Esta técnica permite a criação de estruturas mais estáveis. As cerâmicas feitas pela técnica de paletado mostram uma massa homogênea (Figura $7 b$ ).

Estudos com tomografia e microradiografia de cerâmicas estão em andamento. Resultados preliminares mostram cavidades nas peças de cerâmicas e diferentes tipos de temperos utilizados, sugerindo que a técnica é também promissora para fornecer informações a respeito de tecnologia de fabricação e podem favorecer ainda mais estes estudos.
Figura 7

Neutrongrafias de duas amostras de cerâmica provenientes de um sítio da fase arqueológica Iquiri (A) e outra do sítio Los Angeles (B) mostrando detalhes de manufatura $(A)$ roletado e (B) paletado.

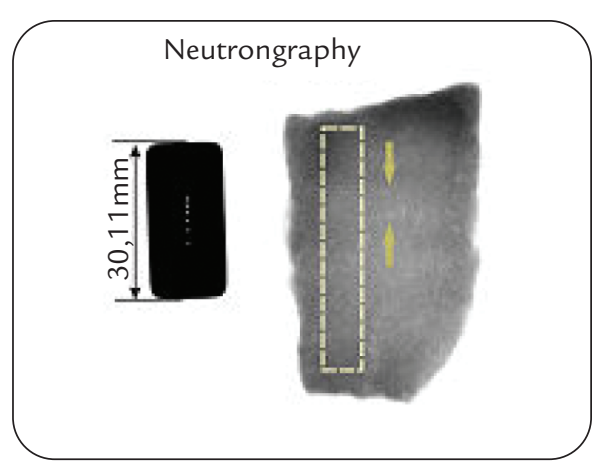

(A)
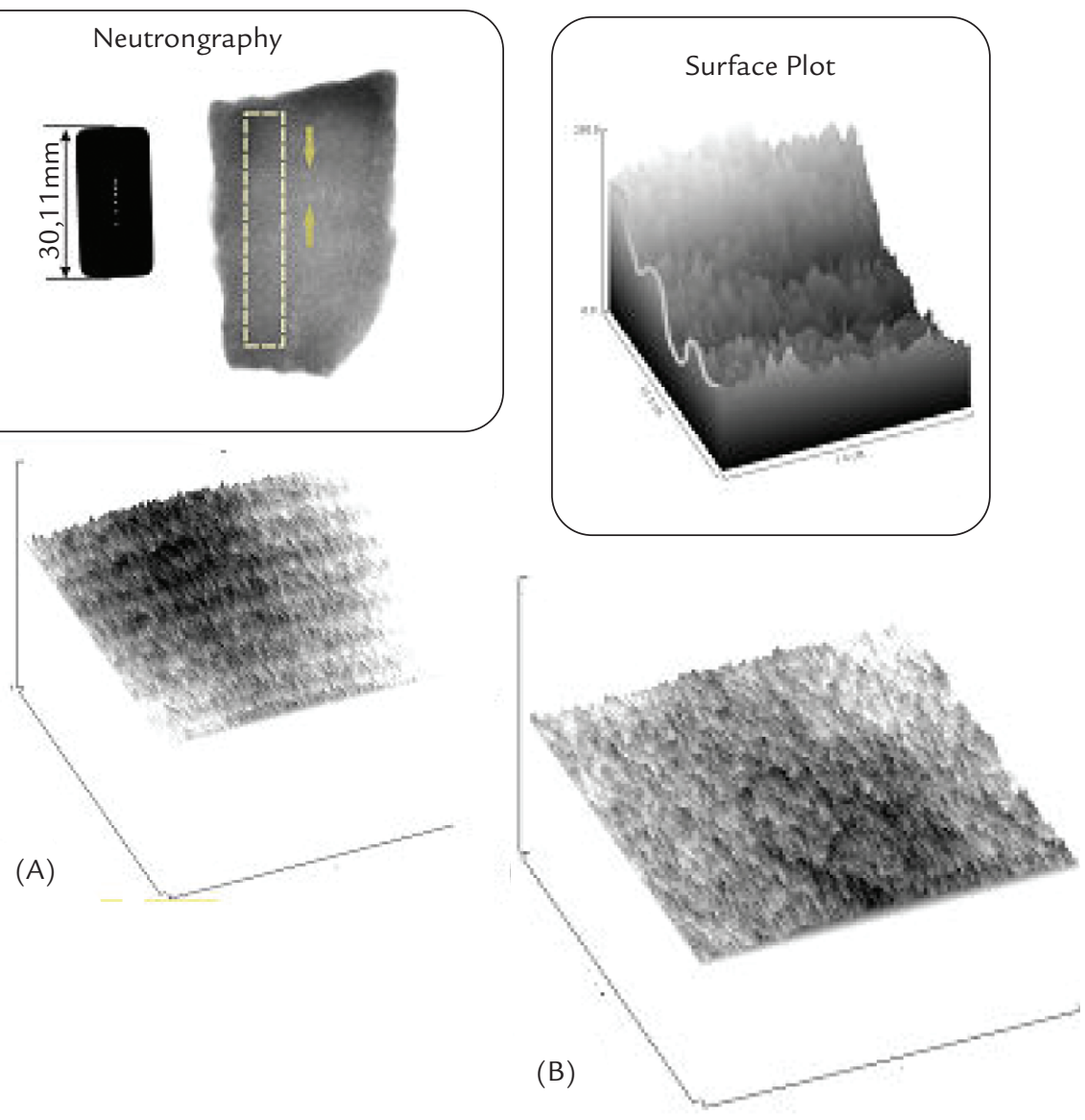


\section{CONCLUSÕES}

Existe uma concordância entre a classificação das fases arqueológicas proposta por arqueólogos e a classificação por composição química nos sítios estudados. As idades obtidas para as cerâmicas dos sítios circulares da bacia Amazônica o Acre sugerem uma direção preferencial do sítio arqueológico Xipamanu I para o sítio Lobão, isto é do sítio mais antigo em direção ao mais recente, e a similaridade das técnicas de fabricação entre as cerâmicas do Lobão e Xipamanu I confirmam isso. Assim, pode-se dizer que a orientação de ocupação do Estado do Acre, por estas culturas, se deu no sentido da Bolívia para a Amazônia. Para resultados mais conclusivos sobre a migração dessas culturas seria necessária a datação de um número maior de amostras de cada um dos sítios associada à análise arqueológica das mesmas. Entre- tanto, as idades obtidas para as cerâmicas encontradas nos sítios com estrutura de terra comprovam a sua relação com a pré-história local. Na Região dos Lagos, Rio de Janeiro os estudos estão ainda em andamento, mas os dados apontam para a contemporaneidade dos sítios em estudo.

Os parâmetros de Mössbauer complementam as informações obtidas pela análise por ativação neutrônica nos estudos de classificação, já que a AAN fornece informações sobre a origem geográfica e o Mössbauer sobre as técnicas de manufatura. A neutrongrafia e a radiografia mostram diferentes detalhes de fabricação, cavidades na massa de argila e diferentes temperos. Resultados preliminares mostram que essas técnicas aplicadas no estudo de produção de cerâmicas fornecem informação sobre sua classificação e tecnologia de fabricação.

\section{REFERÊNCIAS}

Aitken M.J. 1985. Termoluminescence Dating. Academic Press, Londres.

Bellido A.V.B., Latini R.M., Nícoli I.G., Scorzelli R., Solorzano P.M. 2011a. Neutron Activation Analysis and Mössbauer Correlations of Archaeological Pottery from Amazon Basin for Classification Studies. XXXIII Brazilian Workshop on Nuclear Physics, AIP Conference Proceedings, 1351:288-92.

Bellido A.V.B., Latini R.M., Souza M.I.S., Vinagre Filho U.M. 2011b. Manufacturing details by Neutron Radiography of Archaeological Pottery. In: XXXIV Reunião de Trabalho sobre Física Nuclear no Brasil, Foz do Iguaçu.

Bellido A.V.B., Latini R.M., Souza M.I.S. Vinagre Filho U.M. 2011c. Detalles de fabricación por radiografia de neutrones de cerâmicas arqueológicas. 3rth Latin-American Symposium on Physical and Chemical Methods in Archaeology, Art and Cultural Heritage Conservation. Lima.

Bellido A.V.B., Latini R.M., Nícoli I.G.,Neves M.V. 2007. Sítios Arqueológicos com Estruturas Geométricas na Bacia Amazônica: Datação e Caracterização de Cerâmicas. $1^{\circ}$ Simpósio Latino Americano sobre Métodos Físicos e Químicos em Arqueologia, Arte e Conservação de Patrimônio Cultural. São Paulo (SP), Brasil.

Bellido A.V.B. 1989. Neutron Activation Analysis of Ancient Egyptian Pottery. Tese de Doutorado, University of Manchester, Manchester.

Buarque A. 1996. Uma Aldeia Tupinambá em Morro Grande. Coleção Arqueologia, Porto Alegre, 2(1):207-220.

Costa M.L., Carmo M.S., Oliveira E.C., Lima H., Kern D.C., Goeske J. 2009. A mineralogia e composição química de fragmentos de cerâmicas arqueológicas em sítios de terra preta de índio. In: Teixeira W., Madari B., Kern D.C., Benites V., Falcão P.S. (eds.). As Terras Pretas de Índio - caracterização e manejo para a formação de novas áreas. Embrapa.

Dias O. \& Carvalho E. 1988. Arqueo-IAB. Nota técnica do Instituto de Arqueologia Brasileira.

Dias O. \& Carvalho E. 1981. Aspectos da Arqueologia Amazônica. Nota técnica do Instituto de Arqueologia Brasileira.

Gaspar M.D. 1997. A Pré-História do Estado do Rio de Janeiro: Sistemas Sociais Identificados até a Chegada dos Europeus, Rio de Janeiro, Boletim do Museu Nacional do Rio de Janeiro, 60:1-22.

Gilmore G.R. 1983. Technical report Universities Research Reactor.

Latini R.M., Bellido A.V.B., Buarque A. 1999. Estudo de Classificação e Datação de Cerâmicas Arqueológicas do Estado do Rio de Janeiro. In: Congresso Brasileiro de Geoquímica. VII Congresso de Geoquímica dos Países de Lingua Portuguesa, 333-335. 
Latini R.M. 1998. Caracterização, Análise e Datação de Cerâmicas Arqueológicas da Bacia Amazônica através de Técnicas Nucleares. Trabalho de Conclusão de Doutorado. Programa de Pós-graduação em Geociências/Geoquímica Ambiental, Universidade Federal Fluminense.

Latini R.M., Bellido A.V.B. Jr., Dias Junior O. F., Bellido L.F. 2004. Determining the Provenience of Prehistoric Ceramic Artifacts from the Amazon Basin Using Geochemical Tracers. Environmental Geochemistry in Tropical and Subtropical Environments, 1:93-9.

Latini R.M., Bellido A.V.B. Jr., Vasconcelos M.B.A., Dias Junior, O.F. 2001. Classificação de cerâmicas arqueológicas da Bacia Amazônica. Química Nova, 24:724.

McKeever S.W.S. 1985. Thermoluminescence of Solids. University Press, Londres.

Meggers B. 1988. Boletim do Museu Paranaense Emílio Goeldi, Série Antropologia.

Moon P. \& Rodrigues A. 2000. Eram os Deuses Astronautas?. Revista Isto é! edição 1604 de 23 de junho de 2000.

Munayco P., Scorzelli R.B., Latini R.M., Bellido A.V.B. 2011. Estudo de Cerâmicas Arqueológicas da Bacia Amazônica. IX Congresso Ibérico de Arqueologia. Lisboa.

Nícoli I.G. 2000. Estudos de Cerâmicas de Sítios Arqueológicos Circulares da Bacia Amazônica por meio de métodos geoquímicos: Datação e Caracterização. Trabalho de Conclusão de Doutorado. Programa de Pós-graduação em Geociências/Geoquímica Ambiental, Universidade Federal Fluminense.

Tenório M.C.1999. Pré-História da Terra Brasilis, Editora UFRJ, Rio de Janeiro, 376p.

Travesi A. 1975. Analisis por Activacion Neutronica: Teoría, prática y aplicaciones. Junta de Energia Nuclear, Madri, 701 p.

Vinagre Filho U.M., Bellido A.V.B., Latini R.M., Buarque A., Borges A.M. 2008. Estudos de Proveniência em Cerâmicas Arqueológicas Unas do Rio de Janeiro In: XXXI Reunião de Trabalho sobre Física Nuclear no Brasil, São Sebastião (SP), Brasil.

Vinagre Filho U.M., Latini R.M., Bellido A.V.B., Buarque A., Borges A.M. 2005a. Ancient Ceramic Analysis by Neutron Activation in Associate with Multivariate Statistical Methods. Brazilian Journal of Physics, 35:779-781.

Vinagre Filho U.M., Latini R.M., Bellido A.V.B., Buarque A. 2005b. Classification and Provenance Study in ceramics from archaeological sites in Rio de Janeiro state. In: International Nuclear Atlantic Conference - INAC 2005, Santos (SP), Brazil. 